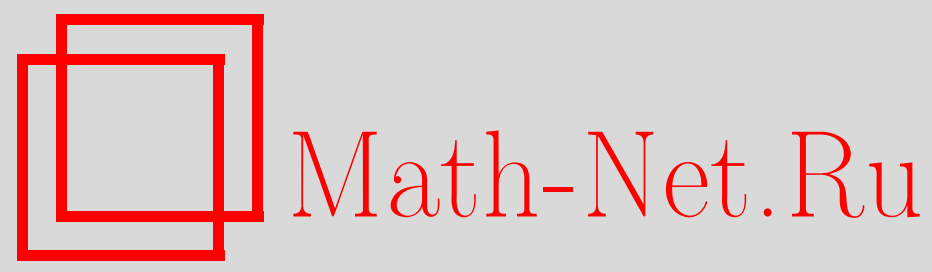

А. А. Пекарский, Наилучшие равномерные рациональные приближения функций посредством ортопроекций, Матем. заметки, 2004, том 76, выпуск 2, 216-225

DOI: https://doi.org/10.4213/mzm101

Использование Общероссийского математического портала Math-Net.Ru подразумевает, что вы прочитали и согласны с пользовательским соглашением http://www.mathnet.ru/rus/agreement

Параметры загрузки:

IP : 54.205 .225 .156

26 апреля 2023 г., $12: 14: 55$ 


\section{НАИЛУЧШИЕ РАВНОМЕРНЫЕ РАЦИОНАЛЬНЫЕ ПРИБЛИЖЕНИЯ ФУНКЦИЙ ПОСРЕДСТВОМ ОРТОПРОЕКЦИЙ}

\section{А. А. Пекарский}

Пусть $C[-1,1]$ - банахово пространство непрерывных комплексных функций $f$ на отрезке $[-1,1]$, наделенных стандартной максимум-нормой $\|f\| ; \omega(\cdot)=\omega(\cdot, f)-$ модуль непрерьвности $f ; R_{n}=R_{n}(f)$ - наилучшее равномерное приближение $f$ посредством рациональных функций (р.ф.) степени не выше $n=1,2, \ldots$ Пространство $C[-1,1]$ рассматривается так же, как предгильбертово относительно скалярного произведения $(f, g)=(1 / \pi) \int_{-1}^{1} f(x) \overline{g(x)}\left(1-x^{2}\right)^{-1 / 2} d x$. Пусть $\mathbf{z}_{n}=\left\{z_{1}, z_{2}, \ldots, z_{n}\right\}-$ набор точек, лежащих вне отрезка $[-1,1]$. Через $\mathscr{F}\left(\cdot, f, \mathbf{z}_{n}\right)$ обозначим ортопроектор, действующий из предгильбертова пространства $C[-1,1]$ в его $(n+1)$-мерное подпространство, состоящее из р.ф.., полюсами которых (с учетом кратности) могут быть лиш точки набора $\mathbf{z}_{n}$. В работе показано, что если $f$ не является р.ф. степени $\leqslant n$, то можно указать набор точек $\mathbf{z}_{n}=\mathbf{z}_{n}(f)$ такой, что

$$
\left\|f(\cdot)-\mathscr{F}\left(\cdot, f, \mathbf{z}_{n}\right)\right\| \leqslant 12 R_{n} \ln \frac{3}{\omega^{-1}\left(R_{n} / 3\right)} .
$$

Библиографория: 11 названий.

1. Постановка задачи. Основной результат и следствия. Пусть $X$ - одно из следуюших банаховых пространств: $C[-1,1]$ - пространство комплексных непрерьвных функций на отрезке $[-1,1] ; C(T)$ - пространство комплексных непрерьвных функций на окружности $T=\{z:|z|=1\} ; C_{A}$ - пространство функций, аналитических в круге $D=\{z:|z|<1\}$ и непрерывных в его замыкании $\bar{D}=D \cup T$. Пространство $X$ наделяется стандартной максимум-нормой $\|\cdot\|=\|\cdot\|_{X} \cdot$

Каждое из пространств $X$ будем рассматривать как предгильбертово. При этом в $C(T)$ и $C_{A}$ скалярное произведение $(f, g)$ функций $f$ и $g$ определяется следующим образом:

$$
(f, g)=\frac{1}{2 \pi} \int_{T} f(z) \overline{g(z)}|d z| .
$$

В пространстве $C[-1,1]$ скалярное произведение определяется так:

$$
(f, g)=\frac{1}{\pi} \int_{-1}^{1} f(x) \overline{g(x)} \frac{d x}{\sqrt{1-x^{2}}}, \quad f, g \in C[-1,1] .
$$

Работа выполнена при финансовой поддержке Российско-Белорусского фонда фундаментальных исследований, грант № 
Через $\mathscr{R}_{n}, n=0,1,2, \ldots$, будем обозначать множество всех рациональных функций (р.ф.) $r$ степени не выше $n(\operatorname{deg} r \leqslant n)$. Пусть $\mathbf{z}_{n}=\left\{z_{1}, z_{2}, \ldots, z_{n}\right\}$ - некоторьй набор точек из расширенной комплекснойплоскости $\overline{\mathbb{C}}$. Через $\mathscr{R}\left(\mathbf{z}_{n}\right)$ обозначим $(n+1)$-мерное линейное пространство р.ф., полюсами которых с учетом кратности могут быть лишь точки множества $\mathbf{z}_{n}$.

Пусть $\mathscr{R}\left(\mathbf{z}_{n}\right) \subset X$. Через $\mathscr{F}\left(\cdot, \cdot, \mathbf{z}_{n}, X\right)$ будем обозначать ортопроектор, действующий из предгильбертова пространства $X$ в его $(n+1)$-мерное подпространство $\mathscr{R}\left(\mathbf{z}_{n}\right)$. Именно, если $f \in X$, то $\mathscr{F}\left(\cdot, f, \mathbf{z}_{n}, X\right) \in \mathscr{R}\left(\mathbf{z}_{n}\right)$ и $f(\cdot)-\mathscr{F}\left(\cdot, f, \mathbf{z}_{n}, X\right) \perp \mathscr{R}\left(\mathbf{z}_{n}\right)$.

Рассмотрим следуюшие примеры. Если $X=C_{A}$ и $z_{k}=\infty$ при $k=1, \ldots, n$, то $\mathscr{F}\left(z, f, \mathbf{z}_{n}, C_{A}\right)$ совпадает с $n$-й частичной суммой ряда Маклорена функции $f$. Если $X=C(T), z_{k}=0$ при $k=1, \ldots, m$ и $z_{k}=\infty$ при $k=m+1, \ldots, 2 m$, то $\mathscr{F}\left(z, f, \mathbf{z}_{2 m}, C(T)\right)$ есть $m$-я частичная сумма тригонометрического ряда Фурье функции $f$. Если $X=$ $C[-1,1]$ и $z_{k}=\infty$ при $k=1, \ldots, n$, то $\mathscr{F}\left(x, f, \mathbf{z}_{n}, C[-1,1]\right)$ совпадает с $n$-й частичной суммой ряда Фурье функции $f$ по многочленам Чебьшева первого рода.

В обшем случае для каждого из пространств $X$ и каждого набора $\mathbf{z}_{n}, \mathscr{R}\left(\mathbf{z}_{n}\right) \subset X$, построены соответствуюшие ортогональные системы р.ф. Именно, для $C(T)$ и $C_{A}$ это сделали Такенака и Мальмквист [1], а для $C[-1,1]$ - Джрбашян и Китбалян [2].

В данной работе мы изучаем порядок равномерных приближений функций из $X$ посредством их ортопроекции на $\mathscr{R}\left(\mathbf{z}_{n}\right)$. При этом полюсы $\mathbf{z}_{n}$ выбираются в зависимости от $f$.

Для $f \in X$ введем $R_{n}=R_{n}(f, X)$ - наилучшее равномерное приближение посредством множества $\mathscr{R}_{n}$, т.е.

$$
R_{n}=R_{n}(f, X)=\inf \left\{\|f-r\|: r \subset \mathscr{R}_{n} \cap X\right\}
$$

Пусть $f$ принадлежит $C[-1,1]$ или $C_{A}$, а $K$ - это отрезок $[-1,1]$ или круг $\bar{D}$ соответственно. В этом случае модулем непрерывности функции $f$ назьвается функция

$$
\omega(\delta)=\omega(\delta, f)=\max \left\{\left|f\left(z_{1}\right)-f\left(z_{2}\right)\right|: z_{1}, z_{2} \subset K ;\left|z_{1}-z_{2}\right| \leqslant \delta\right\}, \quad 0 \leqslant \delta \leqslant 2 .
$$

Если же $f \in C(T)$, то ее модуль непрерывности определяется так:

$$
\omega(\delta)=\omega(\delta, f)=\max \left\{\left|f\left(e^{i t_{1}}\right)-f\left(e^{i t_{1}}\right)\right|: t_{1}, t_{2} \subset \mathbb{R} ;\left|t_{1}-t_{2}\right| \leqslant \delta\right\}, \quad \delta \geqslant 0 .
$$

В последнем случае, очевидно, $\omega(\delta)=\omega(\pi)$ при $\delta \geqslant \pi$.

Как известно (см., например, [3]), функция $\omega(\delta)$ удовлетворяет следующим условиям: $\omega(0)=0, \omega$ непрерьвна и не убьвает на области определения. Пусть $f \neq$ const. Тогда $\omega(\delta)>0$ при $\delta>0$, и мы можем ввести обратную функцию

$$
\omega^{-1}(y)=\min \{\delta \geqslant 0: \omega(\delta)=y\}
$$

Здесь $y$ берется из области значений функции $\delta \mapsto \omega(\delta)$.

Основным результатом данной статьи является следующая 
Tеорема 1. Пусть $f \in X \backslash \mathscr{R}_{n}, n \geqslant 1, \omega(\delta)=\omega(\delta, f) u R_{n}=R_{n}(f, X)$. Тогдa существует набор точек $\mathbf{z}_{n}=\mathbf{z}_{n}(f)$ такой, что

$$
\left\|f(\cdot)-\mathscr{F}\left(\cdot, f, \mathbf{z}_{n}, X\right)\right\| \leqslant 12 R_{n} \ln \frac{3}{\omega^{-1}\left(R_{n} / 3\right)} .
$$

Легко заметить, что правая часть неравенства (1) является бесконечно малой при $n \rightarrow \infty$ лишь в случае, когда выполнено условие Дини, т.е.

$$
\omega(\delta)=o\left(\left(\ln \frac{1}{\delta}\right)^{-1}\right) \quad \text { при } \delta \rightarrow 0 .
$$

Используя теорему 1 и известные верхние оценки $R_{n}(f, X)$ для различных классов функций, можно получить как новые результаты, так и результаты Русака [4], [5] и некоторые результаты автора и Ровбы [6] о порядке наилучших рациональных приближений функций посредством ортопроекции. Ограничимся формулировкой четырех следствий. Необходимые оценки $R_{n}(f, X)$ имеются в [7], [8].

СлЕДСТВИЕ 1. Пусть функиия $f \in C_{A}$ имеет ограниченную вариацию на $T u$ существует $\alpha \in(0,1)$ такое, что $\omega(\delta, f)=O\left(\delta^{\alpha}\right)$ при $\delta \rightarrow 0$. Тогда можно указать последовательность $\mathbf{z}_{n}=\mathbf{z}_{n}(f), n=1,2, \ldots$, для которой

$$
\left\|f(\cdot)-\mathscr{F}\left(\cdot, f, \mathbf{z}_{n}, C_{A}\right)\right\|=o\left(\frac{\ln n}{n}\right) \quad n p u \quad n \rightarrow \infty .
$$

СлЕДСТВИЕ 2. Пусть функиия $f \in C_{A}$ имеет ограниченную вариацию на $T$ и существует $\gamma>1$ такое, что $\omega(\delta, f)=O\left[(\ln (1 / \delta))^{-\gamma}\right]$ при $\delta \rightarrow 0$. Тогда можно указать последовательность $\mathbf{z}_{n}=\mathbf{z}_{n}(f), n=1,2, \ldots$, для которой

$$
\left\|f(\cdot)-\mathscr{F}\left(\cdot, f, \mathbf{z}_{n}, C_{A}\right)\right\|=o\left(n^{-(\gamma-1) / \gamma}\right) \quad \text { npu } n \rightarrow \infty .
$$

СлЕДСТВИЕ 3. Пусть функиия $f \in C[-1,1]$ имеет ограниченную вариацию на $[-1,1]$ и существует $\alpha \in(0,1)$ такое, что $\omega(\delta, f)=O\left(\delta^{\alpha}\right)$ при $\delta \rightarrow 0$. Тогда можсно указать последовательность $\mathbf{z}_{n}=\mathbf{z}_{n}(f), n=1,2, \ldots$, для которой

$$
\left\|f(\cdot)-\mathscr{F}\left(\cdot, f, \mathbf{z}_{n}, C[-1,1]\right)\right\|=O\left(\frac{\ln ^{2} n}{n}\right) \quad n p u \quad n \rightarrow \infty .
$$

СЛЕДСТВИЕ 4. Пусть функиия $f \in C[-1,1]$ имеет ограниченную вариацию на $[-1,1]$ и существует $\gamma>1$ такое, ито $\omega(\delta, f)=O\left[(\ln (1 / \delta))^{-\gamma}\right]$ nри $\delta \rightarrow 0$. Тогда мохсно указать последовательность $\mathbf{z}_{n}=\mathbf{z}_{n}(f), n=1,2, \ldots$, для которой

$$
\| f(\cdot)-\mathscr{F}\left(\cdot, f, \mathbf{z}_{n}, C[-1,1] \|=O\left(n^{-(\gamma-1) /(\gamma+1)}\right) \quad \text { npu } n \rightarrow \infty .\right.
$$

Рациональная аппроксимация посредством ортопроекции класса функций из следствия 1 рассматривалась Русаком [4], [5]. Им получена оценка вида $O\left(\left(\ln ^{2} n\right) / n\right)$.

Нам неизвестно, в каких случаях, оценка (1) будет точной в смысле порядка, а также являются ли точньми оценки из следствий 1-4. В конще п. 2 приводится оценка для пространства $C_{A}$, которая в некоторых случаях является более точной, чем (1) для $X=C_{A}$. Отметим также, что в [6] выделен класс функций, для которых рациональная аппроксимация посредством ортопроекции дает приближение порядка наилучшего. 
2. Схема доказательства теоремы 1. Ортопроекция в пространстве $C_{A}$. Через $L\left(\mathbf{z}_{n}, X\right)$ обозначим норму оператора $\mathscr{F}\left(\cdot, \cdot, \mathbf{z}_{n}, X\right)$, действующего из банахова пространства $X$ на его $(n+1)$-мерноеподпространство $\mathscr{R}\left(\mathbf{z}_{n}\right)$. Иначеговоря, $L\left(\mathbf{z}_{n}, X\right)$ это константа Лебега для соответствующей ортогональной системы. Если $f \in X$, то справедливо неравенство Лебега

$$
\left\|f(\cdot)-\mathscr{F}\left(\cdot, f, \mathbf{z}_{n}, X\right)\right\| \leqslant E\left(f, \mathbf{z}_{n}, X\right)\left(1+L\left(\mathbf{z}_{n}, X\right)\right)
$$

где

$$
E\left(f, \mathbf{z}_{n}, X\right)=\inf \left\{\|f-r\|: r \in \mathscr{R}\left(\mathbf{z}_{n}\right)\right\}
$$

- наилучшее равномерное приближение посредством множества $\mathscr{R}\left(\mathbf{z}_{n}\right)$.

Неравенство (2) играет основную роль при доказательстве теоремы 1 . Идея состоит в выборе полюсов $\mathbf{z}_{n}=\mathbf{z}_{n}(f)$ таким образом, чтобы правая часть (2) была как можно меньше. Ясно, что $E\left(f, \mathbf{z}_{n}, X\right)$ будет минимальным, если в качестве $\mathbf{z}_{n}$ возьмем полюсы дроби наилучшего приближения, т.е. дроби $r^{*} \in \mathscr{R}_{n}$ такой, что $\left\|f-r^{*}\right\|=R_{n}$ (существование $r^{*}$ доказано, например, в [1]). Однако при таком выборе $\mathbf{z}_{n}$ константа Лебега $L\left(\mathbf{z}_{n}, X\right)$ может оказаться очень большой. Поэтому мы выбираем полюсы $\mathbf{z}_{n}=\mathbf{z}_{n}(f)$ так, чтобы $E\left(f, \mathbf{z}_{n}, X\right) \leqslant 2 R_{n}$ и $L\left(\mathbf{z}_{n}, X\right)$ при этом была не слишком большой. Реализация этой идеи наиболее проста в случае пространства $C_{A}$.

Пусть $X=C_{A}$ или $C(T)$ и набор точек $\mathbf{z}_{n}=\left\{z_{1}, z_{2}, \ldots, z_{n}\right\}$ таков, что $\mathscr{R}\left(\mathbf{z}_{n}\right) \subset X$. В этом случае справедлива [9] следующая оценка константы Лебега:

$$
L\left(\mathbf{z}_{n}, X\right) \leqslant 2 \ln \left(1+\sum_{k=1}^{n} \frac{\left|z_{k}\right|+1}{|| z_{k}|-1|}\right)
$$

Здесь при $z_{k}=\infty$ соответствующее слагаемое считается равньм единице.

Нам понадобятся следующие варианты теоремы Джексона (см., например, [3, п. 5.5.4]). Если $f \in C(T)$, то для любого $n=0,1, \ldots$ существует тригонометрический многочлен $u_{n}$ степени не вьше $n$, т.е. рациональная функция вида

$$
u_{n}(z)=\sum_{k=-n}^{n} c_{k} z^{k}, \quad c_{k}=c_{k}(f)
$$

для которого

$$
\left\|f-u_{n}\right\|_{C(T)} \leqslant 3 \omega\left(\frac{1}{n+1}, f\right) .
$$

Если же $f \in C_{A}$, то, как показьвает анализ доказательства из [3], можем считать $c_{k}=c_{k}(f)=0$ при $k=-1,-2, \ldots,-n$. Поэтому, если $f \in C_{A}$, то для любого $n=0,1,2, \ldots$ существует алгебраический многочлен $p_{n}$ степени не вьше $n$, удовлетворяющий неравенству

$$
\left\|f-p_{n}\right\|_{C_{A}} \leqslant 3 \omega\left(\frac{1}{n+1}, f\right) .
$$

В неравенствах (4) и (5) берутся соответствующие модули непрерывности, введенные в п. 1 . 
Лемма 1. Пусть $f \in C_{A}$, причем $f \neq$ const, $\omega(\delta)=\omega(\delta, f), 0<\varepsilon \leqslant \omega(2), r-p . \phi$. такая, что $\|f-r\|_{C_{A}} \leqslant \varepsilon$. Тогда существует р.ф. $r_{\varepsilon}$, все полюсы которой лежсат в области $|z|>1+\omega^{-1}(\varepsilon), \operatorname{deg} r_{\varepsilon}=\operatorname{deg} r u$

$$
\left\|f-r_{\varepsilon}\right\|_{C_{A}} \leqslant 2 \varepsilon
$$

ДокАЗАТЕЛЬСТво. Положим $\rho=\rho(\varepsilon)=1 /\left(1+\omega^{-1}(\varepsilon)\right), f_{\varepsilon}(z)=f(\rho z)$ и $r_{\varepsilon}(z)=$ $r(\rho z)$. Очевидно, $r_{\varepsilon}-$ p.ф., все полюсы которой лежат в области $|z|>1+\omega^{-1}(\varepsilon)$ и $\operatorname{deg} r_{\varepsilon}=\operatorname{deg} r$. Покажем, что (6) также выполнено. Действительно,

$$
\begin{aligned}
\left\|f-f_{\varepsilon}\right\| & =\max _{z \in \bar{D}}|f(z)-f(\rho z)| \leqslant \omega(1-\rho) \leqslant \varepsilon, \\
\left\|f_{\varepsilon}-r_{\varepsilon}\right\| & =\max _{z \in \bar{D}}|f(\rho z)-r(\rho z)| \leqslant \max _{z \in \bar{D}}|f(z)-r(z)|=\|f-r\| \leqslant \varepsilon .
\end{aligned}
$$

Следовательно, $\left\|f-r_{\varepsilon}\right\| \leqslant\left\|f-f_{\varepsilon}\right\|+\left\|f_{\varepsilon}-r_{\varepsilon}\right\| \leqslant 2 \varepsilon$. Лемма 1 доказана.

ДоКАЗАТЕЛЬСТво ТЕОРЕМЫ 1 дЛЯ $X=C_{A}$. Пусть $r^{*} \in \mathscr{R}_{n} \cap C_{A}$ - дробь наилучшего равномерного приближения функции $f$, т.е. $\left\|f-r^{*}\right\|=R_{n}$. Если $\operatorname{deg} r^{*}=n$, то в качестве $\mathbf{z}_{n}=\mathbf{z}_{n}(f)$ возьмем полюсы р.ф. $r_{\varepsilon}^{*}$, построенной согласно лемме 1, при $r=r^{*}$ и $\varepsilon=R_{n}$. Если же $\operatorname{deg} r^{*}=m<n$, то недостающие $n-m$ полюсов будем считать равными $\infty$. Положим $\delta_{n}=\omega^{-1}\left(R_{n} / 3\right)$ и заметим, что $\delta_{n} \leqslant \omega^{-1}\left(R_{n}\right)$. С учетом этого из леммы 1 и неравенств (2) и (3) получим

$$
\left\|f(\cdot)-\mathscr{F}\left(\cdot, f, \mathbf{z}_{n}, C_{A}\right)\right\| \leqslant 2 R_{n}\left[1+2 \ln \left(1+\frac{n\left(2+\delta_{n}\right)}{\delta_{n}}\right)\right]
$$

Согласно $(5) R_{n} \leqslant 3 \omega(1 /(n+1))$ и, следовательно, $\delta_{n} \leqslant 1 /(n+1)$. Поэтому выражение в квадратных скобках (7) не превышает

$$
1+2 \ln \frac{2 n+1}{\delta_{n}} \leqslant 2 \ln \frac{2 \sqrt{e}(n+1)}{\delta_{n}} \leqslant 2 \ln \frac{2 \sqrt{e}}{\delta_{n}^{2}} \leqslant 4 \ln \frac{2}{\delta_{n}} .
$$

Из (7) с учетом последнего неравенства получим (1) для пространства $C_{A}$ с несколько лучшими константами: 8 и 2 вместо 12 и 3 . Теорема 1 для $X=C_{A}$ доказана.

В случае пространства $C_{A}$ наряду с (3) имеет место следующая универсальная оценка (см. [10, теоремы 4.1 и 4.4]) константы Лебега: $L\left(\mathbf{z}_{n}, C_{A}\right) \leqslant 2 n+1$. С учетом (2) находим, что если $f \in C_{A}$, то для любого $n \geqslant 1$ существует набор точек $\mathbf{z}_{n}=\mathbf{z}_{n}(f)$, для которого

$$
\left\|f(\cdot)-\mathscr{F}\left(\cdot, f, \mathbf{z}_{n}, C_{A}\right)\right\| \leqslant 2(n+1) R_{n}\left(f, C_{A}\right) .
$$

Это неравенство иногда дает лучший результат, чем неравенство (1). 
3. Ортопроекция в пространстве $C(T)$. Для $f \in C(T)$ через $F$ обозначим еегармоническоепродолжение в круг $\bar{D}$, т.е. $F$ гармонична в $D$, непрерывна в $\bar{D}$ и $F(z)=f(z)$ при $z \in T$. Как известно, $F$ в открытом круге $D$ представима с помошью интеграла Пуассона:

$$
F\left(\rho e^{i \tau}\right)=\frac{1}{2 \pi} \int_{-\pi}^{\pi} f\left(e^{i(\tau+t)}\right) P_{\rho}(t) d t
$$

где $0 \leqslant \rho<1, \tau \in[-\pi, \pi]$ и

$$
P_{\rho}(t)=\frac{1-\rho^{2}}{1-2 \rho \cos t+\rho^{2}}=1+2 \sum_{k=1}^{\infty} \rho^{k} \cos k t
$$

- ядро Пуассона.

Вомногих работах (см., например, [11]) рассматривалась задача приближения $2 \pi$-периодических функций с помощью ее интеграла Пуассона. Оценка, полученная ниже в лемме 2, является менее точной, чем в [11]. Эта оценка приводится с доказательством ради полноты изложения, а также из-за того, что применение оценки (9) для доказательства теоремы 1 значительно проще, чем применение соответствующей оценки из [11].

Лемма 2. Пусть $f \in C(T) u \omega(\delta)=\omega(\delta, f)$. Тогда при любых $\rho \in[0,1) u$ $\tau \in[-\pi, \pi]$ имеет место неравенство

$$
\left|f\left(e^{i t}\right)-F\left(\rho e^{i t}\right)\right| \leqslant 3 \omega(\sqrt{2(1-\rho)}) .
$$

ДокАЗАТЕЛЬСТво. Поскольку $P_{\rho}(t)>0$ и

$$
\frac{1}{2 \pi} \int_{-\pi}^{\pi} P_{\rho}(t) d t=1
$$

то

$$
\left|f\left(e^{i \tau}\right)-F\left(\rho e^{i \tau}\right)\right| \leqslant \frac{1}{2 \pi} \int_{-\pi}^{\pi}\left|f\left(e^{i \tau}\right)-f\left(e^{i(t+\tau)}\right)\right| P_{\rho}(t) d t .
$$

Сейчас воспользуемся четностью ядра $P_{\rho}(t)$ относительно $t$ и неравенством

$$
\left|f\left(e^{i \tau}\right)-f\left(e^{i(\tau+t)}\right)\right| \leqslant \omega(|t|) .
$$

В результате получим

$$
\left|f\left(e^{i \tau}\right)-F\left(\rho e^{i \tau}\right)\right| \leqslant \frac{1}{\pi} \int_{0}^{\pi} \omega(t) P_{\rho}(t) d t .
$$

Согласно одному из свойств модуля непрерьвности (см., например, [3]) имеет место неравенство

$$
\omega(t) \leqslant \omega(\sqrt{2(1-\rho)})\left(1+\frac{t}{\sqrt{2(1-\rho)}}\right) .
$$

Поэтому

$$
\left|f\left(e^{i \tau}\right)-F\left(\rho e^{i \tau}\right)\right| \leqslant \frac{\omega(\sqrt{2(1-\rho)})}{\pi} \int_{0}^{\pi} P_{\rho}(t) d t+\frac{\omega(\sqrt{2(1-\rho)})}{\pi \sqrt{2(1-\rho)}} \int_{0}^{\pi} t P_{\rho}(t) d t .
$$


Первый из интегралов в (10) равен $\pi$. Для второго интеграла найдем подходящую оценку. С этой целью заметим, что согласно правой части (8) имеет место равенство

$$
\frac{1}{\pi} \int_{0}^{\pi} \sin ^{2} \frac{t}{2} P_{\rho}(t) d t=\frac{1}{2 \pi} \int_{0}^{\pi}(1-\cos t) P_{\rho}(t) d t=\frac{1-\rho}{2} .
$$

Следовательно,

$$
\frac{1}{\pi} \int_{0}^{\pi} t^{2} P_{\rho}(t) d t \leqslant \pi \int_{0}^{\pi} \sin ^{2} \frac{t}{2} P_{\rho}(t) d t=\frac{\pi^{2}}{2}(1-\rho) .
$$

Используя это и неравенство Буняковского-Коши, получим требуемую оценку второго интеграла из (10):

$$
\frac{1}{\pi} \int_{0}^{\pi} t P_{\rho}(t) d t \leqslant\left(\frac{1}{\pi} \int_{0}^{\pi} P_{\rho}(t) d t\right)^{1 / 2}\left(\frac{1}{\pi} \int_{0}^{\pi} t^{2} P_{\rho}(t) d t\right)^{1 / 2} \leqslant \frac{\pi}{2} \sqrt{2(1-\rho)}
$$

Остается подставить в (10) найденные оценки интегралов. Лемма 2 доказана.

Лемма 3. Пусть $f \in C(T), f \neq$ const, $\omega(\delta)=\omega(\delta, f), 0<\varepsilon \leqslant \omega(\pi)$ u p.. $r$ такая, что $\|f-r\|_{C(T)} \leqslant \varepsilon$. Тогда существует р.ф. $r_{\varepsilon}$, удовлетворяющая условиям: $\operatorname{deg} r_{\varepsilon}=\operatorname{deg} r, r_{\varepsilon}$ не имеет полюсов в кольие $\rho \leqslant|w| \leqslant 1 / \rho$, где $\rho=\rho(\varepsilon)=1 /\left(1+\left(\omega^{-1}(\varepsilon / 3)\right)^{2} / 2\right) u$

$$
\left|f(w)-r_{\varepsilon}(w)\right| \leqslant 2 \varepsilon \quad n p u \quad w \in T .
$$

ДокАЗАТЕЛЬСТво. Пусть $F$ и $R$ - гармоническое продолжение функций $f$ и $r$ соответственно с окружности $T$ в круг $\bar{D}$. Тогда функция $|F(z)-R(z)|$ субгармонична в открытом круге $D$ и непрерывна в его замькании. Поскольку $|f(z)-r(z)| \leqslant \varepsilon$ при $z \in T$, то из принципа максимума субгармонической функции получим, что $|F(z)-R(z)| \leqslant \varepsilon$ при $z \in \bar{D}$. В частности, при указанном $\rho=\rho(\varepsilon)$ имеем

$$
|F(\rho w)-R(\rho w)| \leqslant \varepsilon, \quad w \in T
$$

Функция $r(z)$ единственньм образом представима в виде суммы $r(z)=r_{+}(z)+r_{-}(z)$, где $r_{+}$и $r_{-}-$p.ф., причем $r_{+}$не имеет полюсов в $D$, a $r_{-}-$в $\overline{\mathbb{C}} \backslash D$ и $r_{-}(\infty)=0$. Через $R_{+}(z)$ и $R_{-}(z)$ обозначим соответственно гармоническое продолжение $r_{+}$и $r_{-}$с $T$ в $\bar{D}$. Поскольку $r_{+}$аналитична в $\bar{D}$, то $R_{+}(z)=r_{+}(z)$ при $z \in \bar{D}$.

Рассмотрим функцию $R_{-}(z)$ при $z \in \bar{D}$. Пусть, например, $r_{-}(z)=\left(z-z_{0}\right)^{-k}$, где $\left|z_{0}\right|<1$ и $k \in \mathbb{N}$. Легко убедиться, что в этом случае

$$
R_{-}(z)=\frac{|z|^{2 k}}{\left(z-|z|^{2} z_{0}\right)^{k}} \quad \text { при } \quad z \in \bar{D} .
$$

В общем случае для выяснения структуры $R_{-}(z), z \in \bar{D}$, нужно $r_{-}(z)$ разложить в сумму простых дробей и построить гармоническое продолжение для каждой из простых дробей, составляющих $r_{-}(z)$. 
Из равенства $R(z)=R_{+}(z)+R_{-}(z), z \in \bar{D}$, и структуры функций $R_{+}(z)$ и $R_{-}(z)$ для $z \in \bar{D}$ заключаем, что функция $w \rightarrow R(\rho w)$, рассматриваемая лишь на $T$, совпадает с некоторой р.ф. $r_{\varepsilon}(w)$ такой, что $\operatorname{deg} r_{\varepsilon}=\operatorname{deg} r$ и $r_{\varepsilon}$ не имеет полюсов в кольце $\rho \leqslant|w| \leqslant 1 / \rho$.

Покажем, что условие (11) также вьполнено. Действительно, из условия $\|f-r\| \leqslant \varepsilon$ и неравенств $(9),(12)$ получаем, что при $w \in T$ выполняются соотношения

$$
\begin{aligned}
\left|f(w)-r_{\varepsilon}(w)\right| & =|f(w)-R(\rho w)| \leqslant|f(w)-F(\rho w)|+|F(\rho w)-R(\rho w)| \\
& \leqslant 3 \omega(\sqrt{2(1-\rho)})+\varepsilon \leqslant \varepsilon+\varepsilon=2 \varepsilon .
\end{aligned}
$$

Лемма 3 доказана.

ДоКАЗАТЕЛЬСТВо ТЕоРЕМЫ 1 ДЛЯ $X=C(T)$. Пусть $r^{*} \in \mathscr{R}_{n} \cap C(T)$ - дробь наилучшего равномерного приближения функции $f \in C(T) \backslash \mathscr{R}_{n}$. Тогда $R_{n}=\left\|f-r^{*}\right\|>0$. Если $\operatorname{deg} r^{*}=n$, то в качестве $\mathbf{z}_{n}=\mathbf{z}_{n}(f)$ возьмем полюсы р.ф. $r_{\varepsilon}^{*}$, построенной согласно лемме 3 при $r=r^{*}$ и $\varepsilon=R_{n}$. В случае $\operatorname{deg} r^{*}=m<n$ недостающие $n-m$ полюсов будем считать равными $\infty$. Ради краткости положим $\delta_{n}=\omega^{-1}\left(R_{n} / 3\right)$. Из леммы 3 и неравенств $(2),(3)$ находим, что при указанном выборе $\mathbf{z}_{n}$ вьполняется соотношение

$$
\left\|f(\cdot)-F\left(\cdot, f, \mathbf{z}_{n}, C(T)\right)\right\| \leqslant 2 R_{n}\left[1+2 \ln \left(1+\frac{n\left(4+\delta_{n}^{2}\right)}{\delta_{n}^{2}}\right)\right] .
$$

Упростим это оценку. Пусть $m$ - целая часть числа $n / 2$. Согласно (4) имеем

$$
R_{n} \leqslant 3 \omega\left(\frac{1}{m+1}\right) \leqslant 3 \omega\left(\frac{2}{n+1}\right)
$$

Следовательно, $\delta_{n} \leqslant 2 /(n+1)$. С учетом этого выражение в квадратных скобках $(13)$ не превышает

$$
1+2 \ln \frac{4 n+(n+1) \delta_{n}^{2}}{\delta_{n}^{2}} \leqslant 1+2 \ln \frac{4(n+1)}{\delta_{n}^{2}} \leqslant 2 \ln \frac{8 \sqrt{e}}{\delta_{n}^{3}} \leqslant 6 \ln \frac{3}{\delta_{n}} .
$$

Этим теорема 1 для пространства $C(T)$ доказана.

4. Ортопроекция в пространстве $C[-1,1]$. Введем функцию Жуковского

$$
z=\varphi(w)=\frac{1}{2}\left(w+\frac{1}{w}\right)
$$

и ее обратную функцию

$$
w=\psi(z)=z+\sqrt{z^{2}-1} .
$$

Ветвь корня квадратного выбрана так, чтобы $|\psi(z)|>1$ при $z \notin[-1,1]$. При таком выборе ветви мы имеем взаимно конформные отображения областей $\overline{\mathbb{C}} \backslash \bar{D}$ и $\overline{\mathbb{C}} \backslash[-1,1]$. Если границу области $\overline{\mathbb{C}} \backslash[-1,1]$ считать состоящей из двух экземпляров отрезков $[-1,1]$ (верхнего и нижнего), то будем иметь также гомеоморфное соответствие замыканий указанных областей. 
Лемма 4. Пусть $f \in C[-1,1]$, причем $f \neq$ const, $\omega(\delta)=\omega(\delta, f), 0<\varepsilon \leqslant \omega(2)$, $r-p . \phi$. такая, что $\|f-r\|_{C[-1,1]} \leqslant \varepsilon$. Тогда существует $p . \phi . r_{\varepsilon}$, удовлетворяющая условиям: $\operatorname{deg} r_{\varepsilon}=\operatorname{deg} r$, все полюсы $r_{\varepsilon}$ лежстат вне әллипса $|\psi(z)| \leqslant 1+$ $\left(\omega^{-1}(\varepsilon / 3)\right)^{2} / 2 u$

$$
\left\|f-r_{\varepsilon}\right\|_{C[-1,1]} \leqslant 2 \varepsilon
$$

ДокАЗАтЕЛЬСтво. Положим $g(w)=f[\varphi(w)], w \in T$, и $u(w)=r[\varphi(w)], w \in \mathbb{C}$, и применим к этим функциям лемму 3 . В результате получим, что существует р.ф. $u_{\varepsilon}$, удовлетворяющая условиям: $\operatorname{deg} u_{\varepsilon}=\operatorname{deg} u, u_{\varepsilon}$ не имеет полюсов в кольце $\rho \leqslant|\omega| \leqslant 1 / \rho$, где $\rho=\rho(\varepsilon)=1 /\left(1+\left(\omega^{-1}(\varepsilon / 3)\right)^{2} / 2\right)$ и

$$
\left|g(w)-u_{\varepsilon}(w)\right| \leqslant 2 \varepsilon \quad \text { при } \quad w \in T .
$$

Здесь $\omega^{-1}$ - функция, обратная к функции $\omega(\delta)=\omega(\delta, f)$. Замена $\omega^{-1}(\delta, g)$ на $\omega^{-1}(\delta, f)$ при применении леммы 3 законна ввиду неравенства $\omega(\delta, g) \leqslant \omega(\delta, f), \delta \in[0,2]$.

Сделаем в (14) обратную замену $w=\psi(x), x \in[-1,1]$. В результате получим, что

$$
\left|f(x)-u_{\varepsilon}[\psi(x)]\right| \leqslant 2 \varepsilon \quad \text { при } x \in[-1,1] .
$$

Таким образом, нам остается показать, что функция $r_{\varepsilon}(x)=u_{\varepsilon}[\psi(x)]$ является р.ф. степени $\operatorname{deg} r$ и не имеет полюсов в области $|\psi(z)| \leqslant 1 / \rho$.

Пусть все полюсы р.ф. $r$ простые и не равны $\infty$. Общий случай можно получить отсюда с помощью предельного перехода. Очевидно, что оператор перехода от $r$ к $r_{\varepsilon}$ линеен и переводит константы в константы. Поэтому нам достаточно показать, что если $r(x)=1 /\left(x-z_{0}\right), z_{0} \in \overline{\mathbb{C}} \backslash[-1,1]$, то $r_{\varepsilon}(x)$ является р.ф. первой степени с полюсом в области $|\psi(z)|>1 / \rho$.

Положим $w_{0}=\psi\left(z_{0}\right)$. Ввиду соглашения о выборе ветви функции $\psi$ имеем $\left|w_{0}\right|>1$. Несложные вычисления показывают, что

$$
u(w)=r[\varphi(w)]=\frac{1}{\varphi(w)-\varphi\left(w_{0}\right)}=\frac{2 w_{0}^{2}}{w_{0}^{2}-1}\left(\frac{w_{0}}{w-w_{0}}-\frac{1}{w w_{0}-1}\right)
$$

Следовательно, гармоническое продолжение $U(w)$ функции $u(w)$ с $T$ в $\bar{D}$ будет равно

$$
U(w)=\frac{2 w_{0}^{2}}{w_{0}^{2}-1}\left(\frac{w_{0}}{w-w_{0}}-\frac{|w|^{2}}{w w_{0}-|w|^{2}}\right), \quad w \in \bar{D} .
$$

Заменим здесь $w$ на $\rho w$ и будем считать $w \in T$. После несложных преобразований находим

$$
U(\rho w)=\frac{w_{0}^{2}-\rho^{2}}{\rho\left(w_{0}^{2}-1\right)} \cdot \frac{1}{\varphi(w)-\varphi\left(w_{0} / \rho\right)}, \quad w \in T
$$

Следовательно,

$$
r_{\varepsilon}(x)=\frac{w_{0}^{2}-\rho^{2}}{\rho\left(w_{0}^{2}-1\right)} \cdot \frac{1}{x-\varphi\left(w_{0} / \rho\right)}, \quad x \in[-1,1]
$$

Лемма 4 доказана. 
Пусть набор точек $\mathbf{z}_{n}=\left\{z_{1}, z_{2}, \ldots, z_{n}\right\}$ такой, что $\mathscr{R}\left(\mathbf{z}_{n}\right) \in C[-1,1]$. В этом случае аналог неравенства (3) вьглядит так [2]:

$$
L\left(\mathbf{z}_{n}, C[-1,1]\right) \leqslant 2 \ln \left(1+2 \sum_{k=1}^{n} \frac{\left|\psi\left(z_{k}\right)\right|+1}{\left|\psi\left(z_{k}\right)\right|-1}\right) .
$$

Напомним также неравенство Джексона для пространства $C[-1,1]$. Если $f \in C[-1,1]$ и $n=0,1,2, \ldots$, то существует алгебраический многочлен $p_{n}, \operatorname{deg} p_{n} \leqslant n$, для которого

$$
\left\|f-p_{n}\right\|_{C[-1,1]} \leqslant 3 \omega\left(\frac{1}{n+1}, f\right) .
$$

ДОКАЗАТЕЛЬСТВО ТЕОРЕМЫ 1 ДЛЯ $X=C[-1,1]$ проводится по той же схеме, что и для $X=C_{A}$ и $C(T)$. Нужный результат следует из леммы 4 , неравенств $(2),(15)$ и $(16)$. Теорема 1 доказана полностью.

\section{СПИСОК ЦИТИРОВАННОЙ ЛИТЕРАТУРЫ}

[1] Уолш Дж. Л. Интерполяция и аппроксимация рациональными функциями в комплексной области. М.: ИЛ, 1961.

[2] Джрбашян М. М., Китбалян А.А. Об одном обобщении полиномов Чебышева // Докл. АН Арм. ССР. 1964. Т. 37. № 5. С. 263-270.

[3] Тиман А.Ф. Теория приближения функций действительного переменного. М.: ГИФМЛ, 1960.

[4] Русак В.Н. О скорости приближения некоторых классов функций рациональными операторами. Теория приближения функций // Труды Международной конференции по теории приближения функций. Киев, 31 мая-5 июня, 1983. М., 1987. С. 382-386.

[5] Русак В. Н. Рациональные функции как аппарат приближения. Дисс. .. докт. физ.-мат. наук. Киев: 1987.

[6] Пекарский А. А., Ровба Е. А. Равномерные приближения функций Стилтьеса посредством ортопроекции на множество рациональных функций // Матем. заметки. 1999. Т. 65. № 3. С. $362-368$.

[7] Пекарский А. А. Чебышевские рациональные приближения в круге на окружности и на отрезке // Матем. сб. 1987. Т. 133. №1. С. 86-102.

[8] Lorentz G. G., Golitschek M. V., Makovoz Y. Constructive Approximation. Advanced Problems. Berlin: Springer, 1996.

[9] Джрбашян М.M. К теории рядов Фурье по рациональньпм функциям // Изв. АН Арм. ССР. Сер. матем. 1956. Т. 9. № 7. С. 3-28.

[10] Пекарский А. А. Оценки производной интеграла типа Коши с мероморфной плотностью и их приложения // Матем. заметки. 1982. Т. 31. № 3. С. 389-402.

[11] Ульянов П. Л. О приближении функций // Сиб. матем. ж. 1964. Т. 5. № 2. С. 418-437.

Белорусский государственный технологический университет

E-mail: pekarski@bstu.unibel.by 